\title{
À PROPOS DE L'IMAGINAIRE DES MÉDIAS
}

Michel MAFFESOLI ${ }^{*}$ et Moisés de Lemos MARTINS **

Ce numéro de Sociétés réunit un ensemble de textes sur l'imaginaire des médias, qui concrétise une coopération scientifique déjà ancienne entre le Centre d'Études sur l'Actuel et le Quotidien (CEAQ) et le Centro de Estudos de Comunicação e Sociedade (CECS). C'est depuis trois décennies, en effet, que des chercheurs de l'Université Paris Descartes (Sorbonne) et de l'Université du Minho (Braga) mènent ensemble des recherches communes, dont les résultats ont été rendus publics dans des colloques, séminaires et publications.

Les textes qui suivent sont autosuffisants et témoignent d'un phénomène on ne peut plus prospectif : l'émergence d'une culture « cyber » dont ils tentent d'explorer les contours, et ce avec lucidité, originalité et courage. N'est-ce pas le propre d'une authentique pensée que d'inventer un réel qui est, essentiellement, contraire aux vérités usuelles ? On a bien dit réel, ce qui n'est pas réductible à ce principe de réalité avec lequel on le confond fréquemment. De même l'invention, on ne le redira jamais assez, consiste à faire venir au jour (in-venire) ce qui est là. Ce qui est déjà là.

C'est par un effort de volonté, contre les théories convenues, et par une observation intense, contre les évidences intellectuelles, que l'originalité de la pensée rejoint celle de cet être ensemble tout à la fois authentique et souterrain, propre à la vitalité spécifique de ce que l'on a, de tout temps, appelé un état naissant.

C'est bien un tel status nascendi qui est à l'œuuve dans les diverses cybercultures, contaminant, de multiples manières, la vie quotidienne de nos sociétés. C'est, en effet, en termes d'épistémologie qu'il faut poser le problème. Tant il est vrai qu'à

* Professeur des universités ; membre de l'Institut Universitaire de France ; Administrateur du CNRS ; Directeur du CEAQ.michel.maffesoli@ceaq-sorbonne.org

** Professeur de l'Université du Minho, Braga, Portugal ; membre du Centre d'Études de Communication et Société (CECS) ; Directeur des revues scientifiques Comunicação $e$ Sociedade et Anuário Internacional de Comunicação Lusófona ; Président de l'Association Portugaise des Sciences de la Communication (SOPCOM). moisesm@ics.uminho.pt 
bas bruit, mais d'une manière obstinée, les divers médias de communication interactive ont gagné du terrain, et se sont imposés. Tout à la fois pour ce qui concerne les services, les démarches administratives ou bureaucratiques, et dans tous les moments ludiques, où le rêve tend à se substituer à la réalité même.

Souvenons-nous de Thomas Kuhn qui, réfléchissant sur les découvertes scientifiques et leurs conséquences technologiques, a pu montrer comment celles-ci étaient cause et effet de ce qu'il nommait un paradigme. Que l'on peut comprendre comme une matrice qui, en son sens strict, permet l'éclosion d'une nouvelle vie. C'est bien un nouveau paradigme qui est, de nos jours, en gestation et que l'on nomme cyberculture. En lui se rend visible l'hybridité du temps, où l'énergie des machines et les émotions des hommes se mélangent comme dans un amalgame. Et le cyberspace, en tant qu'ambiance de production numérique, constitue autant le voyage que l'on s'attend à faire que l'océan sur lequel il importe de naviguer. L'expérience de la technologie électronique combine, en effet, un passage et une traversée. Nous pouvons faire le passage d'un fleuve d'une marge à l'autre. Le passage nous raconte une expérience contrôlée, maitrisée, sans mystère ni magie, c'est-à-dire aussi sans poésie. Dans les passages nous nous attendons à un voyage sans tourment, tranquille, sans grands obstacles à surmonter. Dans les passages il existe, enfin, l'« habitualité » d'un chemin connu. Bien différente est, cependant, la traversée d'un océan, qui nous impose un tourment permanent, en raison autant de sa nouveauté que de sa dangerosité. La traversée signale le mystère tourbillonnant de la vie, une expérience où l'on surmonte la limite connue, soit de mers, soit de mondes et de connaissances. Et ce nouveau paradigme cyberculturel constitue bel et bien une traversée de ce genre, une traversée vers la nouvelle Amérique d'une nouvelle archive culturelle, qui réactive l'ancienne, l'archaïsme, enfin, la mythologie et, en même temps, reconfigure en permanence la communauté par le désir d'être ensemble.

L'idole, dont il est important de rappeler qu'elle était d'essence féminine, cause et effet de cultes à la " terre mère ", l'idole donc, ne faisait pas appel à la raison, mais au ventre. Il y a quelque chose d'hystérique en elle. C'est l'uterus qui est sollicité. Qu'est-ce que le ventre, sinon le symbole des sens en leur diversité ? Le ventre, en sa position centrale, est le signe de l'entièreté de l'être. Pour utiliser un oxymore instructif, il est vecteur d'une raison sensible, alliant les contraires, les faisant entrer en interaction, en coïncidence.

Au milieu du XIXe siècle, s'est amorcée la révolution optique, par l'invention de la caméra. Ce moment a libéré les images de la cage qui les séquestrait sous une autorité, tant symbolique, d'inspiration judéo-chrétienne, que logocentrique, d'origine gréco-latine. Les images vont alors se multiplier selon un mode profane et séculier, perdant l'aura. Cependant, vers le milieu du XXe siècle, avec le déclenchement de la cybernétique et de l'ordinateur, les images finissent par méduser l'humain. Interagir avec la machine est maintenant une activité expressive, poétique et joyeuse. Bien qu'il soit vrai que la machine agrandit toujours l'espace du contrôle et de la domination, par des processus répétés et mécaniques, il existe 
néanmoins une espèce de syncrétisme où de nouvelles tribus socio-techniques coexistent avec des structures de domination.

La rébellion de l'imaginaire se manifeste avec éclat dans les jeux de rôles, les forums de discussion et les différents blogs et home pages, où la fantaisie, les fantasmes et autres fantasmagories occupent l'essentiel de l'espace et du temps. La raison, la fonctionnalité, l'utilitarisme ne sont pas totalement absents, mais on leur attribue une portion congrue. Ou, plus exactement, par une intéressante inversion de polarité, ils vont servir d'adjuvants à un réel ludique. De maîtres ils deviennent serviteurs. En somme, de la même façon qu'ils réalisent la raison en tant que contrôle, les nouveaux médias électroniques produisent l'effet toujours plus amplifié d'une esthétisation du quotidien.

Il est, à cet égard, instructif de noter combien le festif, l'imaginaire, l'onirique collectifs vont devenir les normes de l'espace « cyber ». Et par là contaminent le territoire privé et la sphère publique. Dans la rationalisation généralisée de l'existence, lorsque se constitue le contrat social, l'on voit s'ériger ce qui fut appelé, familièrement, le « mur de la vie privée ». Sous les coups de boutoir des jeux de rôles et des blogs, ce mur, quand il n'est pas totalement mis à bas, devient pour le moins poreux.

Le " cogito, ergo sum » de Descartes reposait sur une révolution épistémologique d'importance : le fait de penser par soi-même. Et ce dans l'enfermement, dans la forteresse de l'esprit individuel. C'est bien le contraire qui s'exprime sur la "toile», où le partage des images fait que l'on est pensé par l'autre. Que l'on n'existe que par et sous le regard des autres. Ce qui n'est pas sans engendrer ce que l'on peut appeler, pour reprendre une expression de Durkheim, un « conformisme logique».

Voilà bien la reliance qui est en jeu dans tous ces sites. Le mot, d'ailleurs, n'est pas neutre. Le temps se contracte en espace. Il devient un «site " que je partage avec d'autres et à partir duquel je peux " croître ". L'Histoire, avec un grand " $\mathrm{H}$ ", l'Histoire de la modernité, assurée d'elle-même, laisse la place à ces petites histoires, sans réel contenu, mais assurant le lien, permettant du liant. En ces sites communautaires, pour jouer avec l'euphonie des mots, le lieu fait lien. Dans la «parole quotidienne " de cet entretien infini, pour reprendre des mots de Blanchot, se joue, par conséquent, " la profondeur de ce qui est superficiel ». Notre atmosphère est de plus en plus sensitive et libidinale, avec l'émotion, le désir, la séduction et la peau qui deviennent les valeurs les plus répandues de notre culture. La sensibilité et les émotions prennent, en effet, le devant sur les idées et le bios se mélange avec la techné, dans un processus accéléré d'esthétisation générale de l'existence humaine, et toute l'expérience devient « expérience sensible ».

Les réseaux électroniques actuels, dont Hi5, Facebook, Second Life, Twitter, confirment que la parole ne peut pas ne pas nous lier et que l'autre est toujours notre destinée. Dans ces éco-systèmes électroniques s'est produite une hybridation. L'homo sapiens s'est fondu avec l'homo numericus, il s'est fondu avec des logiques socio-techniques. On peut dire, en ce sens, qu'il est arrivé quelque chose de semblable au surgissement de nouvelles espèces animales. Mais peut-être 
devrions-nous parler plus radicalement. Ce processus d'hybridation a commencé avec le langage (l'écriture en tant que proto-histoire, comme on peut dire, rappelant Derrida) ; il a continué par la presse, la photographie (Benjamin) ; le gramophone, le cinéma et la machine à écrire (Kittler); la radio et la télévision (Luhmann); et prend forme aujourd'hui dans cette fusion de bios et de techné, c'est-à-dire d'organique et d'inorganique, dont le cybernaute est une illustration. Le triomphe du langage digital de la communication, grâce à l'échange intensif de messages, photographies, vidéos, informations, nous plonge dans le monde d'une culture hypercommunicative, c'est-à-dire dans «l'extase de la communication » (Baudrillard).

Hi5, Second life, Myspace, Facebook, Twitter, voilà autant de déclinaisons du jeu des images et de la dépense improductive. Tout cela ne sert à rien, mais souligne le prix des choses sans prix. Ces sites sont les formes postmodernes du Potlatch pré-moderne. Ce qui était resté, comme forme résiduelle, dans la sagesse populaire qui « sait » très bien, de savoir incorporé, que parfois qui perd gagne. Et que dans l'inutile de la " tchatche », dans le sans intérêt du blog ou du forum de discussion, voire dans l'obscénité de la " home page ", se (re)trouve la consolidation du lien social.

Quelque chose de sacramentel en quelque sorte. C'est-à-dire rendant visible une force invisible. En ce sens, le virtuel des cybercultures est bien une manière d'exprimer le désir d'être-ensemble. Au travers des frémissements, du grouillement, il saisit les tremblements d'une vie en gestation. On a pu comparer ce nouveau lien spirituel à la " noosphère " du père Teilhard de Chardin. Ce rapprochement n'est pas anachronique, en ce qu'il fait bien ressortir qu'il y a dans les liens invisibles des échanges virtuels quelque chose qui, plus que la dimension économique, plus que la quantification positiviste, plus que les infrastructures matérielles assure une cohésion sociétale dont on n'a pas fini de mesurer les conséquences.

Les joueurs en ligne qui, de Tokyo à Londres, en passant par São Paulo ou Los Angeles, sans oublier telle bourgade reculée des Alpes ou des Carpates, s'épuisent en des joutes interminables, sont reliés par les liens magiques du virtuel. Mais leur réel a une efficacité bien plus forte que les principes de réalité communément admis. Ne serait-ce que parce que leur vie quotidienne est, en son sens fort, déterminée par ces jeux qui les fascinent. lls sont comme aimantés par des polarités lointaines et invisibles. C'est bien cela la noosphère du virtuel de la cyberculture.

Le " mystère " est ce qui unit des initiés entre eux, ceux partageant les mêmes mythes. Mais qu'est-ce qui a fait que, dans la floraison de ces cultes, et alors qu'ils avaient des spécificités assez proches, seul le christianisme ait survécu ? Certes, les raisons doivent en être multiples. On va en privilégier une. Comme un corps sécrétant ce qui permet sa survie, les petites sectes chrétiennes vont sécréter le dogme de la communion des saints. Unissant les morts aux vivants et ceux-ci entre eux.

Ainsi, la communauté de Rome est-elle unie, en esprit, à celle de Lyon, de Narbonne, de Braga, de Milan. Ainsi se crée, en pointillé, une union qui va donner naissance à une Église d'importance et à une culture dont toute l'Europe est issue. 
Grâce à cette " communion ", un commerce va s'établir entre les diverses églises locales. Échanges et partages constituant un corpus mysticum tirant toutes les conséquences doctrinales et organisationnelles de la reliance dont il a été question.

Revenons à ce qui est en train de se passer sous nos yeux. Même processus initiatique, mêmes échanges et partages de tous ordres. Le peer to peer est à l'ordre du jour en de nombreux domaines. De même, c'est par contamination électronique que se développent les phénomènes altermondialistes, la diffusion des informations, les rassemblements frivoles ou sérieux. Un terme traduit bien tout cela : flashmob, la mobilisation instantanée.

Même dans l'ordre de la connaissance, avec les grossières erreurs et méfaits que l'on sait, Wikipédia tient le haut du pavé, symbole, s'il en est, que le savoir ne vient plus du haut, qu'il n'émane plus d'un pouvoir vertical, mais se répand à l'image de la puissance de base, d'une manière horizontale.

Ce ne sont là que quelques indices de la cyberculture naissante. Le développement technologique qui avait participé de la démagification du monde et contribué à l'isolement des individus, à ce que l'on peut appeler la grégaire solitude, s'inverse en son contraire, et contribue à une nouvelle reliance : être, toujours, en contact, en union, en communion, être branché.

Oui, c'est bien une nouvelle culture qui s'élabore avec Internet, la nouvelle Amérique d'une nouvelle archive culturelle.

Le "cyberspace » est un lien, aux contours indéfinis, infinis, où, d'une manière matricielle, s'élabore la rencontre avec l'autre, où se conforte le corps social. $\mathrm{Ne}$ peut-on pas dire, de ce fait, qu'il constitue la communion des saints postmoderne? 